\title{
MORBILIDAD DE LA ENFERMEDAD PULMONAR OBSTRUCTIVA CRÓNICA EN COLOMBIA. RESULTADOS DEL ESTUDIO SANEPOC-2
}

\section{MORBIDITY OF CHRONIC OBSTRUCTIVE PULMONARY DISEASE IN COLOMBIA. RESULTS OF THE STUDY SANEPOC-2}

Isaac Kuzmar ${ }^{1,5}$, Carlos Eduardo Giraldo Ospina ${ }^{2}$, Germán Oved Acevedo Osorio ${ }^{3}$, Guido Rua Salas ${ }^{4}$.

\section{Resumen:}

Objetivo: relacionar las morbilidades de los pacientes pertenecientes al programa de EPOC de una institución de salud del municipio de Anserma, Caldas (Colombia). Material y Métodos: estudio descriptivo de 51 pacientes a quienes les fueron tomadas espirometrías exploratorias, los hallazgos fueron contrastados con las principales comorbilidades registradas en la Historia Clínica a través de estadística descriptiva e inferencial. Resultados: $50 \%$ presentaron al menos una comorbilidad registrada, principalmente la Hipertensión Arterial seguida de la Enfermedad Renal Crónica, se encontró un comportamiento asociado entre la clasificación GOLD y la presencia de síntomas con valores no estadísticamente significativos; no se encontró asociación estadísticamente significativa entre las comorbilidades y los valores espirométricos. Conclusiones: se hacen necesarias nuevas investigaciones que valoren de forma clara la causalidad en la población colombiana.

Palabras clave: EPOC; morbilidades; espirometría; Colombia; síntomas

\section{Abstract:}

Objective: to relate the morbidities of patients belonging to the COPD program of a health institution in Anserma, Caldas (Colombia). Material and Methods: a descriptive study was presented with a sample of 51 patients who underwent exploratory spirometry, the findings were contrasted with the main commodities reported by the patients or the Medical Record through descriptive and inferential statistics. Results: $50 \%$ of the evaluated population had at least one recorded comorbidity; the main morbidity found was Arterial Hypertension followed by Chronic Kidney Disease; an associated behavior was found between the GOLD classification and the presence of symptoms with non-statistically significant values; no statistically significant association was found between comorbidities and spirometric values. Conclusions: new research is needed that clearly values causality in the Colombian population.

Keywords: COPD; Morbidities; spirometry; Colombia; symptoms.

\footnotetext{
1 Profesor-Investigador. Facultad de Ciencias de la Salud. Departamento de Investigación. Universidad Simón Bolívar, Colombia. 2 Médico Cirujano Universidad de Caldas, Epidemiólogo Fundación Universitaria del Área Andina

3 Instrumentador Quirúrgico Fundación Universitaria del Área Andina, Epidemiólogo Fundación Universitaria del Área Andina

4 Médico Cirujano Universidad Metropolitana de Barranquilla, Epidemiólogo Fundación Universitaria del Área Andina

5 Email de contacto: isaac.kuzmar@unisimonbolivar.edu.co
} 


\section{Introducción}

De acuerdo a los lineamientos planteados en las guías GOLD se define Enfermedad Pulmonar Obstructiva Crónica (EPOC) como una patología común prevenible y tratable caracterizada por la limitación persistente al flujo del aire que usualmente es progresiva y se encuentra asociada a una respuesta inflamatoria crónica de la vía aérea y del pulmón a agentes irritantes ${ }^{1}$. Hoy por hoy se considera esta patología dentro del grupo de enfermedades crónicas no trasmisibles ${ }^{2}$, con un comportamiento en su prevalencia que ha evidenciado un ascenso constante como la cuarta causa de muerte a nivel mundial ${ }^{3}$.

Lo naturaleza creciente de la enfermedad ha precipitado el desarrollo de diferentes estrategias para medir el comportamiento epidemiológico de la EPOC. Estrategias como PLATINO ${ }^{4}$ y BOLD 5 han tratado de unificar criterios para determinar la prevalencia disminuyendo la variabilidad del interobservador ${ }^{6}$ en los diagnósticos de EPOC obteniendo resultados estimados entre el 8-10\% mundial ${ }^{7}$.

Estudios como el ECLIPSE ${ }^{8}$ han dado pie a cuestionamientos sobre el comportamiento natural de la enfermedad y han puesto en tela de juicio el papel de la VEF1/FVC como medida para la estadificación de la enfermedad, no obstante la espirometría continua siendo el gold estándar para el diagnóstico de la EPOC 9 .

En Colombia, el estudio PREPOCOL ${ }^{10}$ determinó una prevalencia aproximada del 8,9\% para la enfermedad tras haber estructurado un estudio de corte trasversal donde se valoró por espirometria y por cuestionario validado una muestra de pacientes con factores de riesgo para EPOC en 5 ciudades del territorio nacional (Bogotá, Medellín, Cali, Bucaramanga y Barranquilla); en la ciudad de Medellín se encontró una prevalencia parcial del 13,5\%; por su localización geográfica se toma como referente para la región ubicada en el eje cafetero, no obstante la publicación es clara al no recomendar extrapolaciones de la prevalencia de la enfermedad de un área geográfica a otra.

El Hospital San Vicente de Paul ESE realizó un primer estudio que buscó determinar la carga de la enfermedad pulmonar obstructiva crónica en el programa de enfermedades crónicas no trasmisibles al que llamó SANEPOC-1, reportando como conclusión principal el subdiagnóstico de la EPOC con un promedio del $2.07 \%$ de pacientes con diagnóstico por espirometría, el estudio concluyó la necesidad de nuevos estudios con mayor complejidad que apunten a la determinación de la prevalencia y la caracterización de pacientes con cuadro de EPOC en la institución y el municipio.

El objetivo del estudio SANEPOC-2 es determinar la carga de morbilidad asociada a la enfermedad pulmonar obstructiva crónica a través de un estudio observacional de corte trasversal en el servicio de enfermedades crónicas no trasmisibles y el programa de EPOC en una institución hospitalaria de primer nivel.

\section{Material y Métodos}

El presente estudio busca determinar la carga de morbilidad asociada a la enfermedad pulmonar obstructiva crónica a través de los análisis espirométricos los pacientes pertenecientes al programa de enfermedad pulmonar obstructiva crónica del municipio de Anserma, Caldas.

Tipo de estudio: estudio de corte trasversal, descriptivo, prospectivo.

Sujetos: Se utiliza una muestra de 572 pacientes con diagnóstico de EPOC del municipio de Anserma Caldas pertenecientes al programa de EPOC (nivel de confianza 85\%; Heterogeneidad 50\%).

Criterios de inclusión: Pacientes mayores de 40 años, Pacientes pertenecientes al programa de Enfermedad Pulmonar Obstrucitva Crónica (EPOC) y que acepten ingresar en la investigación.

Criterios de exclusión: Pacientes con datos incompletos en la historia clínica, pacientes con patología mental de base, pacientes que no puedan realizar la prueba espirométrica: antecedente de cirugía toracoabdominal en los últimos 3 meses, antecedente de síndrome coronario agudo en los últimos tres meses, antecedente de desprendimiento de retina o proceso quirúrgico oftalmológico en los últimos tres meses, hospitalización por causas cardíacas en los últimos 3 meses, terapia antituberculosa activa, gestación positiva, frecuencia cardíaca mayor a 160 latidos por minuto.

Criterios para diagnóstico del aumento en el diámetro anteroposterior(AP): diámetro AP de igual o mayor longitud (en centímetros) respecto al diámetro trasverso de la caja torácica asociado a los datos detectados por el evaluador en la inspección (tórax en tonel). 
Criterios para diagnóstico de cianosis: Coloración azulada en zonas distales del cuerpo (dedos, nariz, pabellones auriculares entre otros) visualizada por el evaluador y asociada a una oximetría de pulso inferior a $85 \%$.

Componente bioético: la presente investigación comprende una intervención de riesgo mínimo, se contó con el consentimiento informado de los pacientes y con los permisos respectivos de la institución.

Recolección de datos y plan de análisis: los datos serán recolectados de forma complementaria, una parte a través de la valoración clínica y espirométrica de los pacientes incluidos en el protocolo de investigación; como complemento se asocian datos de la historia clínica extraídos de la base de datos de la institución la cual es alimentada por los datos provistos de cada uno de los pacientes seleccionados; el total de la información fue tabulada en el programa Microsoft Excel 2013. Para el análisis de los datos se utilizó estadística descriptiva e inferencial, se utilizó el programa Stata versión XII. Se considera nivel de significación para $\mathrm{p}<0,05$.

\section{Resultados}

Los datos internos del programa de Enfermedad Pulmonar Obstructiva Crónica evidencian un total de 572 pacientes (tabla 1) de los cuales 60(10,48\%) corresponden a pacientes oxigenorequirientes; del $100 \%$ de los pacientes pertenecientes al programa, el 64,3\% se encuentra bajo la figura diagnóstica de EPOC sin estratificar; el $20,1 \%$ bajo la figura de pacientes con EPOC exacerbado y el 15,6\% se encontró bajo la condición de EPOC con infección aguda de la vía aérea inferior. Se convocó a 81(14,16\%), de los cuales $20(24,69 \%)$ declinaron su opción de participación en el estudio; y del total restante $(n=61)$ se excluyó el $16,39 \%$ tras filtrar por criterios de eliminación y exclusión; los detalles de la selección se discriminan en la figura 1.

Tabla 1: Muestra de pacientes con diagnóstico de EPOC

\begin{tabular}{|ccccc|}
\hline E.P.S. & \multicolumn{2}{c}{ TOTAL PACIENTES } & \multicolumn{2}{c|}{ Oxigenorequirientes } \\
\hline Caprecom & 91 & $15.9 \%$ & 16 & $26.7 \%$ \\
Cafesalud & 279 & $48.77 \%$ & 28 & $46.7 \%$ \\
Asmetsalud & 157 & $27.44 \%$ & 10 & $16.7 \%$ \\
Contributivo & 45 & $7.89 \%$ & 6 & $9.9 \%$ \\
\hline Total & 572 & $100 \%$ & 60 & $100 \%$ \\
\hline
\end{tabular}

Se encontró una carga de morbilidad total en el 50\%(tabla 2) de los pacientes valorados, al respecto se evidenció un predominio en patologías de origen cardiovascular siendo la hipertensión arterial y la enfermedad renal crónica las patologías que aportaron mayor carga respecto al total; al valorar la coexistencia de estas dos enfermedades se encontró que el 95,4\% de los pacientes con hipertensión arterial cursaron con algún grado de enfermedad renal crónica; el 100\% de los pacientes que cursaban con al comorbilidad diabetes fueron hipertensos y el 94,1\% compartían las morbilidades hipertensión arterial y dislipidemia.

Tabla 2: Distribución carga de morbilidad por sexo y ubicación.

\begin{tabular}{|cccccc|}
\hline \multirow{2}{*}{ Características Morbilidades } & \multicolumn{2}{c}{ Distribución Sexo } & Distribución Ubicación & Porcentaje \\
\cline { 2 - 5 } & Hombre & Mujer & Rural & Urbano & Total \\
\hline Carga de Morbilidad * & $50 \%$ & $50 \%$ & $37,0 \%$ & $63,0 \%$ & $50,0 \%$ \\
Hipertensión Arterial & $54,2 \%$ & $45,8 \%$ & $62,5 \%$ & $37,5 \%$ & $47,1 \%$ \\
Enfermedad Renal Crónica & $50 \%$ & $50 \%$ & $63,6 \%$ & $36,4 \%$ & $43,1 \%$ \\
Dislipidemia & $41,2 \%$ & $58,8 \%$ & $82,4 \%$ & $17,6 \%$ & $33,3 \%$ \\
Cardiopatía Coronaria & $71,4 \%$ & $28,6 \%$ & $57,2 \%$ & $42,8 \%$ & $13,7 \%$ \\
Diabetes Mellitus & $40 \%$ & $60 \%$ & $80 \%$ & $20 \%$ & $9,8 \%$ \\
Asma & $75 \%$ & $25 \%$ & $75 \%$ & $25 \%$ & $7,8 \%$ \\
Hipotiroidismo & $66,7 \%$ & $33,3 \%$ & $66,7 \%$ & $33,3 \%$ & $5,9 \%$ \\
Enfermedad & $100 \%$ & $0 \%$ & $0 \%$ & $100 \%$ & $2,0 \%$ \\
Cerebrovascular & $56,4 \%$ & $43,6 \%$ & $69,3 \%$ & $30,7 \%$ & $76,5 \%$ \\
Asistencia a Controles & $60,8 \%$ & $39,2 \%$ & $62,7 \%$ & $37,3 \%$ & $50 \% * *$ \\
\hline Total & & & & \\
\hline
\end{tabular}

* Carga de morbilidad: al menos una patología crónica asociada; ** corresponde al porcentaje global de carga de morbilidad 
Respecto a la Distribución geográfica pese a encontrarse un mayor porcentaje de pacientes pertenecientes a la cabecera municipal se encontró una relación de pacientes rurales:urbanos de 2:1 para patologías como hipertensión arterial, enfermedad renal crónica, hipotiroidismo y patología coronaria; llama la atención un aumento en la relación de 4:1 para los pacientes con diagnóstico de dislipidemia, pese a ello se encontró una adherencia a controles de 7:3 en la población analizada; los detalles de la carga de morbilidad se encuentran

Al valorar el comportamiento de los valores espirométricos respecto a la presencia de comorbilidades (tabla 3) se encontró una disminución en los valores reportados en las espirometrías no obstante dichos cambios no fueron consistentes al momento de agrupar los pacientes respecto al género; los resultados evidenciados tras los análisis multivariados evidenciaron relaciones que no fueron estadísticamente significativas. El valor medio para CVF en los pacientes valorados fue de 73,78 (IC 65,$75 ; 81,82 p=0,47$ ); resultados similares para la variable VEF1:73,45(IC 65,54; $81.36 \mathrm{p}=0,47$ ) ambos con valoraciones no significativas tras los análisis estadísticos.

Tabla 3: Relación Carga de Morbilidad y variables Espirométricas

\begin{tabular}{|c|c|c|c|c|}
\hline \multirow{2}{*}{ Relación Morbilidades } & \multicolumn{2}{|c|}{ Capacidad Vital Forzada } & \multicolumn{2}{|c|}{$\begin{array}{c}\text { Volumen Espiratorio } \\
\text { Forzado en 1 segundo }\end{array}$} \\
\cline { 2 - 5 } & $\mathbf{p}$ & Intervalo de Confianza & $\mathbf{p}$ & Intervalo de Confianza \\
\hline Carga de Morbilidad * & 0,275 & $-9,08 ; 31,12$ & 0,252 & $-8,20 ; 30,45$ \\
Hipertensión Arterial & 0,019 & $6.63 ; 68.61$ & 0,019 & $6.61 ; 69,02$ \\
Enfermedad Renal Crónica & 0,606 & $-39,32 ; 23,23$ & 0,633 & $-38,99 ; 23,98$ \\
Dislipidemia & 0,654 & $-29,26 ; 18,57$ & 0,601 & $-30,36 ; 17,79$ \\
Cardiopatía Coronaria & 0,174 & $-43,62 ; 8,17$ & 0,078 & $-49,45 ; 2,71$ \\
Diabetes Mellitus & 0,364 & $-42,46 ; 15,90$ & 0,329 & $-43,77 ; 14,99$ \\
Asma & 0,864 & $-31,72 ; 37,63$ & 0,900 & $-32,73 ; 37,10$ \\
Hipotiroidismo & 0,181 & $-13,64 ; 70,14$ & 0,779 & $-36,29 ; 48,07$ \\
Enfermedad Cerebrovascular & 0,127 & $-141,12 ; 18,23$ & 0,386 & $-115,0 ; 45,43$ \\
Asistencia a Controles & 0,741 & $-24,94 ; 17,86$ & 0,619 & $-25,69 ; 15,46$ \\
\hline
\end{tabular}

Al valorar la presencia de disnea durante la intervención se encontró que el $90 \%$ de los pacientes que cumplían criterios para disnea se encontraron entre un estadio Gold de 2-3 con una distribución 1:2 $(p=0,001)$, el $100 \%$ de los pacientes que cumplieron criterios para cianosis cursaban con un Gold 4 $(\mathrm{p}=0,001)$ al momento de la valoración; respecto al aumento antero posterior del tórax durante la intervención se encontró que el $80 \%$ de los pacientes se agrupo alrededor de los estadios 2-3 con una distribución de 3:1 no obstante dada la limitada cantidad de casos no se considera un resultado concluyente $(p=0,18)$; los comportamientos de los síntomas respecto a los valores espirométricos se ilustran en la figura 2, llama la atención la acentuación de las alteraciones en el género femenino.

\section{Discusión}

Con el presente estudio se buscó aproximar a través de espirometrías la posible relación entre las morbilidades de los pacientes con diagnóstico de enfermedad pulmonar obstructiva crónica pertenecientes al programa de EPOC de una institución de salud del municipio de Anserma Caldas; de acuerdo a los resultados encontrados la hipótesis alterna planteada por el equipo investigador no pudo ser validada, hecho que puede ser explicado por el diseño trasversal del presente estudio asociado a un tamaño de muestra reducido que limita la estructuración de asociaciones desde el punto de vista estadístico.

Frente a la carga de morbilidad hallada en los pacientes valorados se encontraron resultados concordantes con Sievi y colaboradores ${ }^{11}$ quienes a través de un estudio de corte trasversal reportaron como primera comorbilidad en frecuencia el diagnóstico de hipertensión arterial (50\%), de forma estrecha 
se asoció la enfermedad coronaria y la presencia de dislipidemia, concluyendo que la coexistencia entre 1 a 5 comorbilidades podrían impactar sobre el desarrollo de la enfermedad $(p<0,001)$ postulando dichos resultados como potenciales predictores en la disminución de la actividad física en la enfermedad; de forma complementaria dicha disminución, valorada a través de la caminata de los 6 minutos ha mostrado ser un predictor claro de mortalidad de acuerdo a los resultados planteados por el Índice BODE tanto para mortalidad por cualquier causa como para mortalidad por causas pulmonares (riesgo relativo para mortalidad por todas las causas: $1.34 \mathrm{IC}(1,26-1,42) \mathrm{P}<0,001)^{12}$; de forma complementaria TorresSánchez y colaboradores en una revisión sistemática de la literatura encontraron múltiples publicaciones que relacionan la presencia de deterioro cognitivo y Epoc entre 3-30\% de acuerdo a las publicaciones analizadas, no obstante dada la naturaleza de la investigación no es posible valorar causalidad en los hallazgos reportados ${ }^{13}$; al respecto un estudio trasversal realizado en la ciudad de bello Horizonte por el grupo de Pimienta-Pedrosa encontró potenciales relaciones entre la disminución en la puntuación del índice de Charlson y la presencia de EPOC en pacientes con diagnóstico previo de síndromes Demenciales ${ }^{14}$.

La presente investigación no logró establecer relaciones claras entre las morbilidades reportadas y los comportamientos espirométricos encontrados, datos concordantes de forma parcial con la literatura mundial, Black-Shinn y colaboradores valoraron la asociación entre hipertensión arterial - síndrome de falla cardíaca y EPOC evidenciándola presencia de enfermedad cardiovascular del $15 \%$ precediendo al EPOC tras realizar ajuste por elementos confusores categorizaron ambas variables como independientes respecto al EPOC, reforzando la necesidad de nuevos estudios que valoren causalidad ${ }^{15}$; en otros aspectos Hersh y colaboradores ${ }^{16}$ a través de un estudio donde valoraron la presencia de enfisema, valorado a través de tomografía computarizada y diabetes mellitus encontraron potenciales asociaciones estadísticamente significativas; por su parte Torres - Sánchez ${ }^{17}$ en una revisión sistemática de la literatura encontró múltiples publicaciones donde se reportaron asociaciones entre patologías endoncrinometabólicas y el desarrollo de EPOC; Calverley y colaboradores en el estudio TORCH encontró una prevalencia del $27 \%$ de fallecimientos por causas cardiovasculares en pacientes con EPOC $^{18}$; se requieren nuevos estudios que cuenten con una metodología que permita establecer asociaciones y causalidades entre la enfermedad y sus comorbilidades más frecuentes para la población colombiana.

Tras Valorar la presencia de síntomas de acuerdo a la clasificación GOLD y espirométrica de los pacientes se encontraron comportamientos que sugieren una relación entre la clasificación Gold, la espirometría y la presencia de síntomas, no obstante deben considerarse como factores limitantes el tamaño de muestra y la potencia de inferencia que los datos pueden suministrar al igual que el método exploratorio utilizado dado que las valoraciones espirométricas fueron exploratorias, no se realizaron pruebas pre y post broncodilatador; este hecho abre la necesidad de nuevas investigaciones que utilicen la prueba espirométrica tal como lo exige el Gold Estándar al igual que una medición de síntomas más refinada haciendo uso de escalas validadas y baremadas; los elementos antes enunciados pueden explicar las discordancias evidenciadas respecto a los resultados publicados en el estudio ECLIPSE ${ }^{19}$.

El presente estudio cuenta con limitaciones, por ser un estudio exploratorio no se pueden establecer relaciones de causalidad entre la variables que se trabajaron, se requieren estudios prospectivos que evalúen a fondo este objetivo; si bien hubo resultados que fueron concordantes entre síntomas y valores espirométricos el tamaño de la muestra no permite establecer asociaciones claras, hecho que lleva a interpretar los resultados con cautela.

\section{Conclusiones}

Aunque hay un $50 \%$ de morbilidades asociadas a la enfermedad pulmonar obstructiva crónica en el municipio de Anserma Caldas; los resultados de la presente investigación no son concluyentes respecto a la aproximación causal entre comorbilidades y patrones espirométricos; por lo que nuevas investigaciones con diseños metodológicos destinados de forma específica a valorar asociaciones causales entre comorbilidades y valores espirométricos son requeridas; de igual manera el desarrollo de estudios longitudinales en población colombiana que validen las potenciales asociaciones por establecer.

\section{Agradecimientos}

A Isabel Cristina Bermudez por su gestión en la logística requerida para la presente investigación; a Victoria Cajas Bravo por su colaboración en la recolección de los datos. 


\section{Bibliografía}

1. Vestbo J, Hurd SS, Agusti AG, Jones PW, Vogelmeier C, Anzueto A, Barnes PJ, Fabbri LM, Martinez FJ, Nishimura M, Stockley RA, Sin DD, Rodriguez-Roisin R: Global strategy for the diagnosis, management, and prevention of chronic obstructive pulmonary disease: GOLD executive summary. Am J Respir Crit Care Med 2012, 187(4):347-365.

2. Menezes Ana y cols. PROYECTO LATINOAMERICANO
OBSTRUCCIÓN PULMONAR. Libro oficial. Capítulo 1. Www.platino-alat.org

3. WORLD HEALTH STATISTICS2014. WORLD Cataloguing-in-Publication Data. ISBN 9789240692671 (PDF)

4. AnaMBMenezes, Cesar G Victora, Rogelio Perez-Padilla and the PLATINO Team. The Platino project: methodology of a multicenter prevalence survey of chronic obstructive pulmonary disease in major Latin American cities. BMC Medical Research Methodology 2004, 4:15 doi:10.1186/1471-2288-415

5. Buist Sonia McBurnie Mary Ann et al. International variation in the prevalence of COPD(The BOLD Study): a population-based prevalence studyLancet 2007; 370: 741-50

6. Araya Marcela et al. Calidad de las espirometrías en un estudio epidemiológico de terreno. Factores determinantes de la necesidad de repetir el examen en el estudio Platino-Chile. Rev Chil Enf Respir 2005; 21: 155-163Rev Chil Enf Respir 2005; 21: 155-163Rev Chil Enf Respir 2005; 21: 155-163Rev Chil Enf Respir 2005; 21: 155-163Rev Chil Enf Respir 2005; 21: 155-1

7. Buist Sonia McBurnie Mary Ann et al. International variation in the prevalence of COPD (The BOLD Study): a population-based prevalence study Lancet 2007; 370: 741-50

8. VestboJ.Anderson W. et al.Evaluation of COPD Longitudinallyto Identify Predictive SurrogateEnd-points (ECLIPSE).EurRespir J 2008; 31: 869-873DOI: 10.1183/09031936.00111707CopyrightERS Journals Ltd 2008

9. American Thoracic Society. Standardization of spirometry, 1994 update. Am J Respir Crit Care Med 1995; 152:1107- 1136

10. Caballero Andres et al. Prevalence of COPD in Five ColombianCities Situated at Low, Medium, andHigh Altitude (PREPOCOL Study)*.CHEST/133/2/ FEBRUARY, 2008

11. Noriane a. Sievi, oliver senn, thomas brack et al.Impact of comorbidities on physical activity in COPD. Respirology (2015) 20, 413-418 (c) 2015 Asian Pacific Society of Respirology

12. Bartolome R. Celli, Claudia G. Cote, Jose M. Marin et al. The Body-Mass Index, Airflow Obstruction, Dyspnea, and Exercise Capacity Index in Chronic Obstructive Pulmonary Disease. $n$ engl j med 350;10 www.nejm.org march 4, 2004

13. Irene Torres-Sánchez, Elisabeth Rodríguez-Alzueta, Irene Cabrera-Martos et al. Cognitive impairment in COPD: a systematic review. J Bras Pneumol. 2015;41(2):182-190. http://dx.doi.org/10.1590/S1806-37132015000004424

14. Fausto Aloísio Pedrosa Pimentaa, Maria Aparecida Camargos Bicalhob, Marco Aurélio Romano-Silva.Chronic diseases, cognition, functional decline, and the Charlson index in elderly people with dementia. REV ASSOC MED BRAS. 2013;59(4):326-334

15. Black-Shinn, Kinney GL, Wise AL, Regan EA, Make B y cols. Cardiovascular disease is associated with COPD severity and reduced functional status and quality of life. COPD. 2014 Sep;11(5):546-51. doi: 10.3109/15412555.2014.898029. Epub 2014 May 15.

16. Craig P Hersh1, Barry J Make, David A Lynch, et al.Non-emphysematous chronic obstructive pulmonary disease is associated with diabetes mellitus. Hersh et al. BMC Pulmonary Medicine 2014, 14:164 http://www.biomedcentral.com/1471-2466/14/164

17. Irene Torres-Sánchez, Marie Carmen Valenza, Fernando Carrasco et al. Alteraciones endocrinometabólicas en la enfermedad pulmonar obstructiva crónica. Nutr Hosp. 2013;28(4):1022-1030 ISSN 0212-1611 • CODEN NUHOEQ S.V.R. 318

18. Calverley P, Anderson J, Celli B, Ferguson G, Jenkins $C$, Jones $P$ et al. Salmeterol and Fluticasone Propionate and Survival in Chronic Obstructive Pulmonary Disease. N Engl J Med 2007; 356: 775-89.

19. Vestbo J, Anderson W, Coxson HO, Crim C, Dawber F, Edwards L, Hagan G, Knobil K, Lomas DA, MacNee W, Silverman EK, Tal-Singer R, ECLIPSE Investigators: Evaluation of COPD Longitudinally to Identify Predictive Surrogate End-points (ECLIPSE). Eur Respir J 2008, 31(4):869-873. 